\title{
A new method for detecting cross-inhibition effects in the environmental biocatalytic processes
}

\author{
ZOLTÁN HERKE $^{1 *}$, THOMAS MASKOW ${ }^{1}$, ZSOLT ISTVÁN NÉMETH $^{2}$ \\ ${ }^{1}$ UFZ, Helmholtz Centre for Environmental Research, Department of Environmental, Leipzig, Germany \\ ${ }^{2}$ Institute for Chemistry, Faculty of Forestry, University of West Hungary, Sopron, Hungary
}

\begin{abstract}
Biocatalytic processes based on the use of different microbial seed cultures and enzyme mixtures are of steadily increasing interest for cleaning contaminated sites. Commercially available "biocatalysts" acting on various target pollutant mixtures have an extremely wide range of application. The situation is aggravated by the fact that these "biocatalysts" often consist of a badly defined mixture of microorganisms and enzymes. The reaction rate and efficiency of biodegradation processes depend on several variables (e.g. bioavailability of nutrients and terminal electron acceptors, $\mathrm{pH}$, temperature, composition of the contamination, or water activity). In addition, an important influencing factor is the inhibition of key enzymes by the composition of the reaction mixture (pollutants) of the contaminated site. The inhibitors often change the reaction kinetics in a very complex way. This paper describes a study on a new method that allows to identify and describe the inhibition effects easily and fast, without resorting to any specific enzyme kinetic evaluating software. This issue is especially important because the method should also be adaptable for badly defined "biocatalysts". The method utilizes latent information in the biocatalytic kinetic data sets. It condenses the complex degradation behavior which is a simple linear relation providing the information about the occurrence and strength of potential inhibition effects. Furthermore, principal component analysis also proved to serve information about the inhibition effects. The applicability of our evaluation strategy has been successfully confirmed using the experimental results as well as model calculations.
\end{abstract}

Key words: bioremediation, inhibition effect, modeling, regression analysis, principal component analysis (PCA)

\section{Introduction}

Bioremediation treatments are environmental technologies in which environmental contamination is reduced by microorganisms and/or higher organisms (plants, animals) or a mix of enzymes obtained from them (Karigar and Rao, 2011). For cleaning contaminated sites, the successful application of mixtures of allochthonous microorganisms and enzymes (hereafter referred as "biocatalysts") has been reported (Vidali, 2001; Sutherland et al., 2004). The metabolic breakdown of several organic contaminant compounds was mapped, and the role of enzymes involved in these processes is defined.

The success of biocatalytic decontamination depends strongly on the influence of the site-specific properties on the activity of the mixture of applied allochthonous microorganisms and enzymes. The bioconversion parameters (the efficiency of metabolism - how big will be the environmental risk of the products - and the degra- dation rate) are affected by the properties of pollutants as well as the properties of the contaminated site (soil structure and quality, $\mathrm{pH}$, temperature, water activity, bioavailability of oxygen, other terminal electron acceptors, and mineral nutrients) (Dua et al., 2002; Dana and Bauder, 2011).

Nowadays, enzymatic bioremediation has become an attractive and easily feasible alternative to systems using whole bacteria or other organisms. The growth of microorganisms may be influenced by many factors such as $\mathrm{pH}$, temperature, oxygen, soil structure, and so on. Therefore, the use of enzymatic mixtures is much simpler and easier to predict. The most suitable enzymes for remediation processes are i.e. those involved in ligninmetabolism, e.g. laccases and lignin-peroxidases from white-rot fungi or some bacterial, intracellular enzymes like mono- or dioxygenases. The mechanisms of action of most enzymes used in bioremediation processes are 
well known (Alcalde et al., 2006; Karigar and Rao, 2011; Megharaj et al., 2011). However, many companies sell mixtures of microorganisms and enzymes as biocatalysts for cleaning contaminated sites. In such case, it is difficult to predict capabilities and limitations of the use of such systems.

It has been suggested in the literature that microbial or enzymatic degradation of a pollutant can be strongly influenced by the presence of other compounds in the mixture. Often negative effects such as competitive inhibition have been reported (Reardon et al., 2000; Brandt et al., 2004, Lin et al., 2007). Therefore, in order to ensure effective execution of bioremediation, it is necessary to explore the possible inhibition mechanisms. In addition to this, the companies selling mixtures of microorganisms and enzymes as biocatalysts conceal the composition of the mixture. Therefore, using these products in the presence of various pollutant mixtures, such as BTEX or chlorinated hydrocarbon compounds, the possibility of the inhibition effects' appearances is fairly realistic.

The main objective of our work was to create a data evaluation method to detect the presence or absence of the inhibition effect on such complex biocatalytic systems. In our bioconversion experiments, we used two homologous compounds such as benzaldehyde and toluene. By applying this simple method, it is not elementary for the calculation of the kinetic parameters' $\left(K_{M}, r_{\max }\right)$ alteration to explore the inhibition effects or using other specific software, such as DynaFit or VisualEnzymics.

\section{Materials and methods}

\section{Simulation of the biodegradation processes}

The easiest case of the turnover of a pollutant $S$ (in $\mathrm{mmol} / \mathrm{l})$ into a product $P$ (in $\mathrm{mmol} / \mathrm{l})(v-$ stoichiometry coefficient) is described in equation 1 .

$$
S \rightarrow v P
$$

The reaction kinetics is often well described by the Michaelis-Menten equation (2) with the parameter $r_{\max }$ (in $\mathrm{mmol} /(\mathrm{l} \cdot \mathrm{h})$ ) and $K_{M}($ in $\mathrm{mmol} / \mathrm{l}$ ).

$$
\frac{d S}{d t}=-\frac{1}{v} \frac{d P}{d t}=-r_{\max } \frac{S}{K_{M}+S}
$$

The simplest case is when one component inhibits the biodegradation of another one. Our model assumes inhibition in the biocatalytic conversion by mixtures of benzaldehyde and toluene. Typical kinetic parameters of the single compounds $\left(K_{M}\right.$ and $\left.r_{\max }\right)$ can be taken from literature (Trigueros et al., 2010; Peper et al., 2011). The bioconversion kinetics of both compounds in a mixture is described by the differential equation system (3).

$$
\begin{aligned}
\frac{d S^{B}}{d t} & =-r_{\max }^{B} \frac{S^{B}}{K_{M}^{T}\left(1+\frac{S^{T}}{K_{i}}\right)+S^{B}} \\
\frac{d S^{T}}{d t} & =-r_{\max }^{T} \frac{S^{T}}{K_{M}^{T}+S^{T}}
\end{aligned}
$$

The differential equation system (3) contains one equation for a competitive inhibition (the upper). The inhibition parameter is $K_{i}(\mathrm{in} \mathrm{mmol} / \mathrm{l})$. The superscript (B and T) stands for benzaldehyde and toluene, respectively. Kinetic data series of the pollutants $(S=S(t))$ were generated by the ODE solver included in SCILAB 5.5.1 software (Scilab Enterprises, Versailles, France). Numerous other inhibition models are conceivable and applicable, but the competitive inhibition is easily exploitable, and therefore eligible for our study. The parameters are depicted in Table 1.

\section{Experimental methods}

\section{Materials}

Benzaldehyde and toluene were obtained from Merck (Germany), $\mathrm{HgCl}_{2}$ and the other chemicals of analytical grade were obtained from Sigma-Aldrich (Germany). The biocatalyst (Bioclean) was produced by a Hungarian enterprise (Malatechwater Ltd., Budapest, Hungary). This product is a protein mixture consisting of different enzymes and bacteria. It is usually applied for a technical breakdown of hydrocarbon pollutants. The initial concentrations of benzaldehyde and toluene were $3.628 \mathrm{mmol} \cdot 1^{-1}$ and $2.300 \mathrm{mmol} \cdot 1^{-1}$, respectively. The biodegradations of benzaldehyde and toluene were investigated individually and in mixtures in which $3 \mathrm{ml}$ of the biocatalyst was added to the aquatic solution $(7 \mathrm{ml})$ of pollutants.

The bioconversions of the pollutants were quantified using gas chromatography (Agilent HP GC-HS 6890 gaschromatograph with G1888 Headspace sampler and an OPTIMA delta $3(60 \mathrm{~m} \times 0.32 \mathrm{~mm} \times 0.1 \mu \mathrm{m})$ column, Santa Clara, CA). The enzymatic or microbial reactions were stopped by adding $4 \mathrm{mM} \mathrm{HgCl}_{2}$ after 13, 24, 32, 40, and $48 \mathrm{~h}$, and samples were taken for later analysis. 
Table 1. Initial parameters of the model decomposition of the benzaldehyde and toluene substrates

\begin{tabular}{l|c|c|c|c}
\hline \multicolumn{1}{c|}{ Model substrates } & $r_{\max }\left(\mathrm{mmol} \cdot 1^{-1} \cdot \mathrm{h}^{-1}\right)$ & $S_{0}\left(\mathrm{mmol} \cdot 1^{-1}\right)$ & $K_{M}\left(\mathrm{mmol} \cdot 1^{-1}\right)$ & $K_{i}\left(\mathrm{mmol} \cdot 1^{-1}\right)$ \\
\hline BENZALDEHYDE & 0.045 & 3.628 & 1.817 & - \\
\hline TOLUENE & 0.020 & 2.300 & 1.150 & - \\
\hline BENZALDEHYDE_INH1 & 0.045 & 3.628 & 1.817 & 0.009 \\
\hline BENZALDEHYDE_INH2 & 0.045 & 3.628 & 1.817 & 0.047 \\
\hline BENZALDEHYDE_INH3 & 0.045 & 3.628 & 1.817 & 0.090 \\
\hline
\end{tabular}

\section{Statistical analysis}

The data from the model and experimental curves were subjected to testing the monotonicity's alteration of the kinetic curves with and without competitive inhibition effects. The generated and experienced inhibition effect was visualized via simple regression and principal component analysis (PCA). Therefore, the SCILAB 5.5.1 software (Scilab Enterprises, Versailles, France) was used. The analysis of covariance was performed using StatsDirect 2.8 software (StatsDirect Ltd, Altrincham, UK).

\section{Results and discussion}

To illustrate the effect of a competitive inhibition, a simulation of enzymatic bioconversion was performed by solving the differential equation system (3). The results shown in Figure 1 demonstrate that the substrate kinetic curves were modified significantly by the competitive inhibition effect.

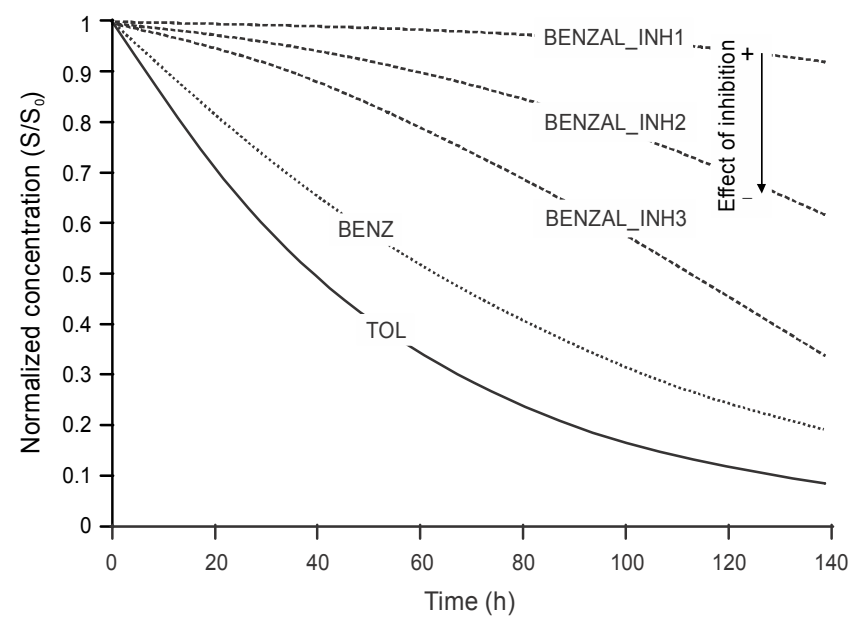

Fig. 1. Normalized kinetic curves of model pollutants as a function of time. BENZAL and TOL stand for benzaldehyde and toluene, respectively. The kinetic curves were calculated without inhibition. BENZAL_INH1-2-3 show inhibition effects of varying strength due to the interaction with toluene.

$K_{i}$ is increasing from 1 to 3
The experiments were performed to characterize the biodegradation of single and mixture pollutants. The normalized experimental concentration data are exemplarily shown in Figure 2. Fitting kinetic curves to substrate concentrations was necessary to interpolate the experimental data. Interpolated "quasi" experimental data are required for the regression and PCA.

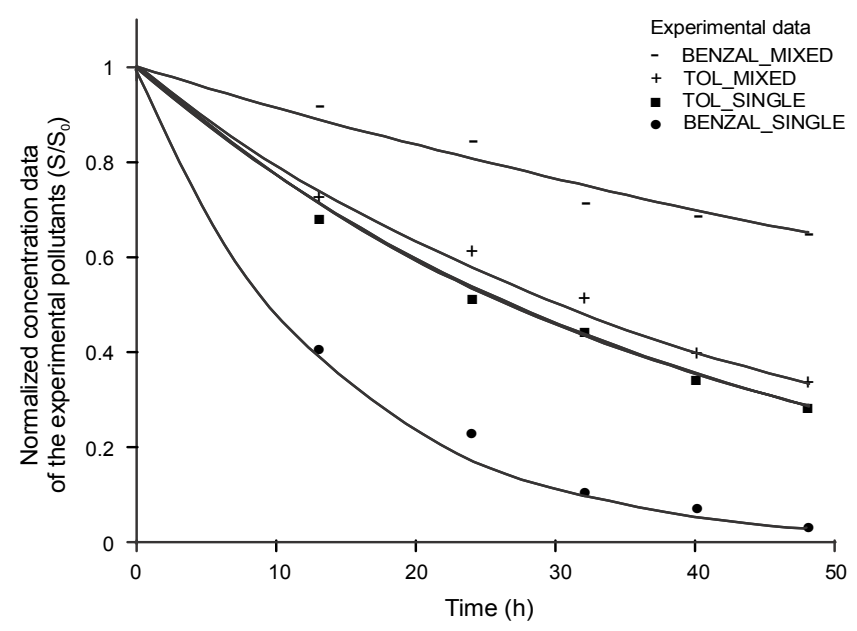

Fig. 2. Kinetic curves fitted on experimental concentration data. The experimental concentrations are represented by markers and fitting curves by solid lines. "TOL_SINGLE" and "BENZAL_SINGLE" stand for the biodegradation data of single benzaldehyde and toluene here as "TOL_MIXED" and "BENZAL_MIXED" indicate degradation data of mixtures

\section{Regression analysis of model kinetic curves}

The applied biocatalyst is capable of breaking down benzaldehyde as well as toluene. Using a plot of normalized concentrations against each other is helpful to indicate the presence or absence of the potential inhibition effects. Figure 3 shows such a plot. If degradation of both pollutants follows the same kinetic model (e.g. Michaelis-Menten), then approximately linear relationships are expected (Fig. 3A, SINGLE). If one of the pollutants follows another kinetic model (e.g. competitive inhibi- 
A

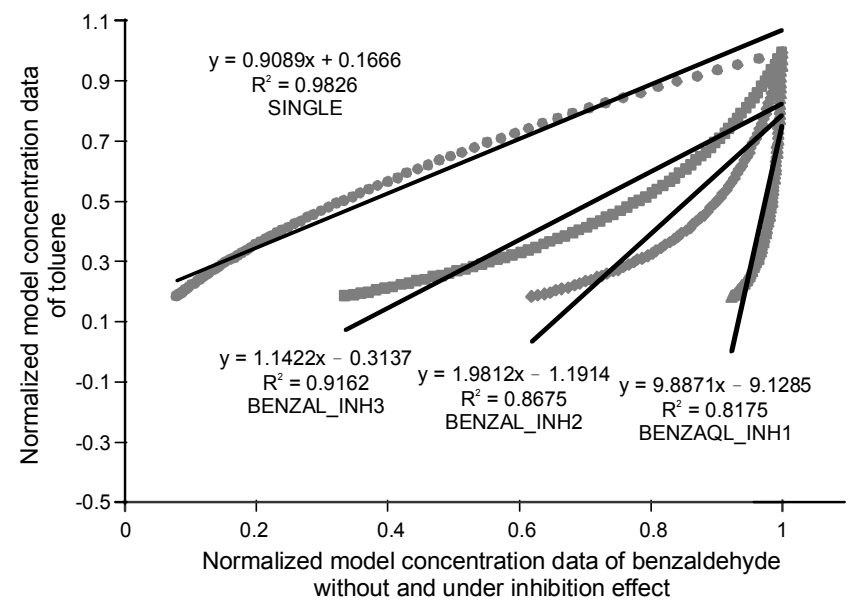

B

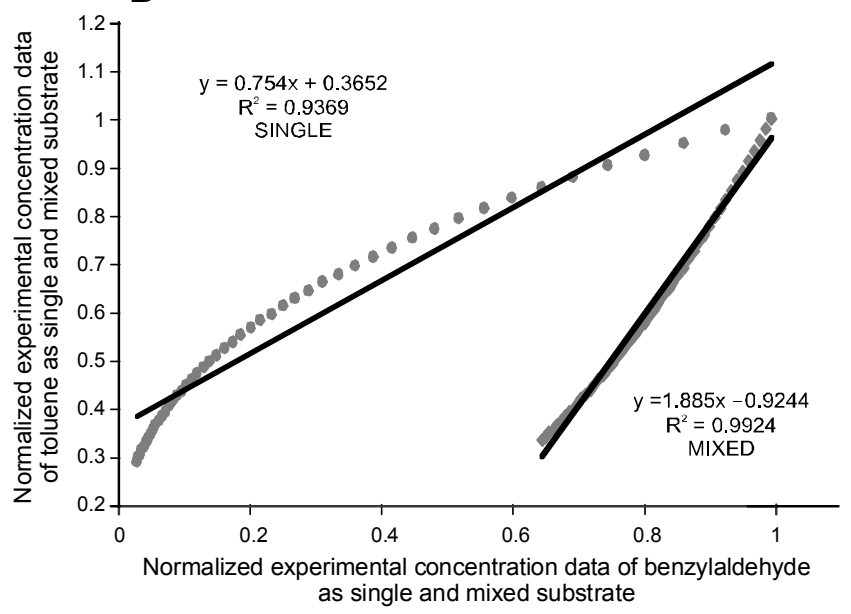

Fig. 3. Correlations between the normalized concentration data of toluene vs. benzaldehyde: A) model data without inhibition (SINGLE) and under various intensity of competitive inhibition effects (BENZAL_INH1-3); B) experimental data of independent degradation trials with single substrate degradation (SINGLE) and of experiments with mixed substrates (MIXED)

A

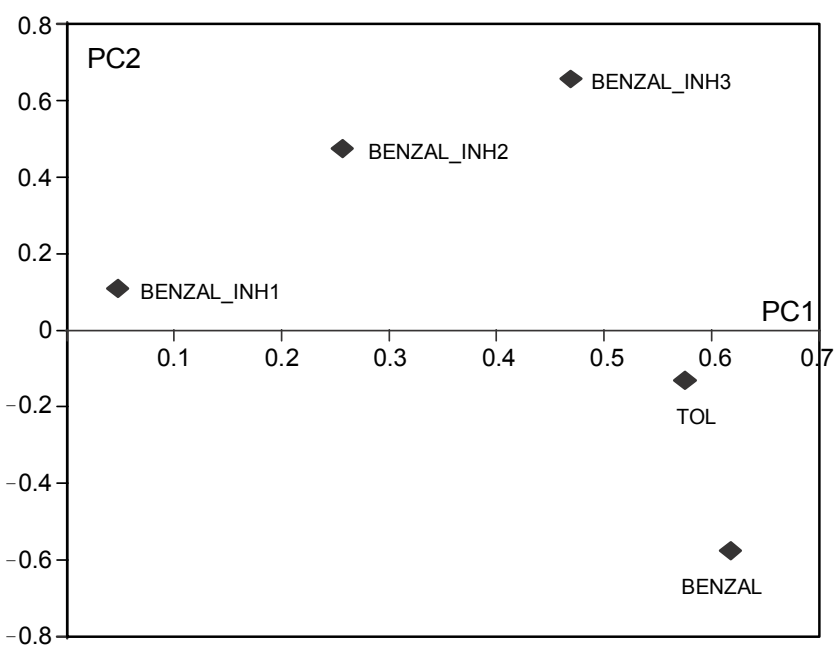

B

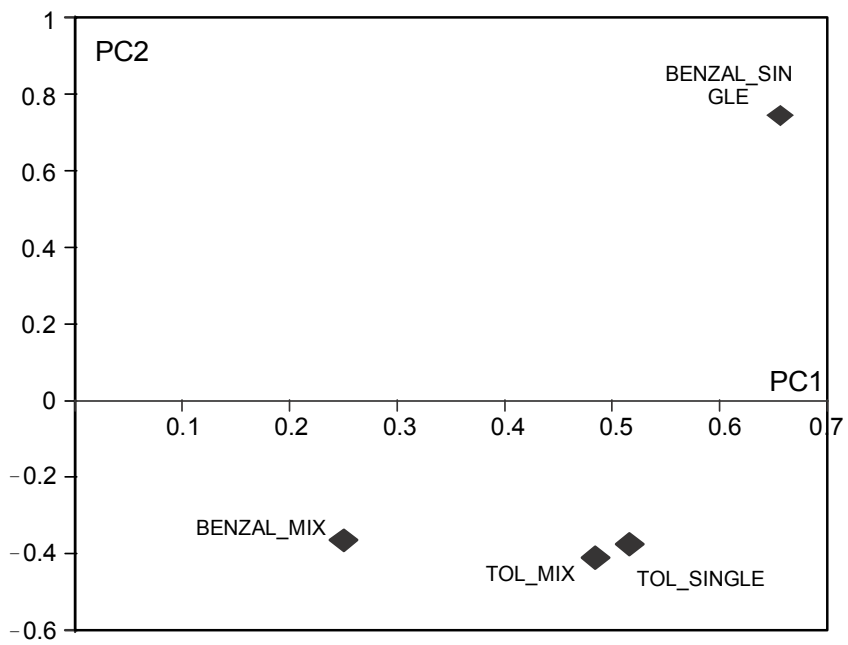

Fig. 4. PCA of model (A) and experimental (B) biodegradation data: A) stands BENZAL and TOL for kinetics without inhibition and BENZAL_INHI1-3 for inhibition of different strength; B) stand BENZAL_SINGLE and TOL_SINGLE for the degradation of single benzaldehyde and single toluene, respectively, whereas BENZAL_MIX and TOL_MIX stand for the biodegradation of mixtures thereof

tion), then the linearity vanishes and the curve shape changes. In such a case, a question emerges whether the linearity (regression coefficient), the slope of a linear data trend, or the intercept may be taken as a measure of inhibitory effects. This thesis is based on the fact that linear regressions of complex data are relatively simple and straightforward. The linear regression has the advantage of being capable to detect the potential inhibition effects in a very fast and simple way. It is important to emphasize that in our approach, the alteration of the linear regression parameters (intercept and slope) was in the focus and not the goodness-of-fit of the model. For this reason, linear regression was applied in all cases; however, nonlinear relationships between the data series are suggested.

Figure $3 \mathrm{~A}$ shows that the linear regression parameters are changed significantly by the competitive inhibition of toluene. The slopes of the regressions are incre- 
ased while the intercepts are decreased by the inhibition effect with respect to the reference curve. Similar trends were also observed in the case of the other two inhibition effects. Following this pattern, the alteration of the regression parameters is influenced by the strength of the competitive inhibition.

\section{Regression analysis of experimental kinetic curves}

The thesis, in which inhibition effect can be indicated by linear regression, was verified by treating the experimental data of the biodegradation of benzaldehyde and toluene, similarly. The regression lines of benzaldehyde and toluene experimental data series are depicted in Fig. 3B. The variation of regression parameters is in accordance with the model results which suppose an inhibition effect between benzaldehyde and toluene. A significant deviation in the linear relationship between kinetic curves in model, as well as experimental results, is rendered by a covariance analysis (data are not presented herein).

\section{PCA of model and experimental kinetic curves}

The data matrix, created from kinetic curves of substrate concentrations, can also be investigated by PCA to visualize the monotonicity's alteration of the kinetic curves. The columns of the matrix contain the concentrations of toluene and benzaldehyde while the rows represent the respective reaction times. In consequence of this data representation, the most important information is about the kinetic curves' appearance into only the first two vectors of the projection $(\mathrm{P})$ matrix. In view of this kind of data set-up, the analysis omits the evaluation of the score (T) matrix. Details of the performed PCA can be taken from Esbensen et al. (2002). In this way, it is possible to establish some pattern in shifting the points of various substrate concentrations under inhibition from the loading plot. The different substrates or substrate mixtures, taking part in various kinds of biodegradation process, are represented by specific points on the loading plot of $\mathrm{PC} 1$ and $\mathrm{PC} 2$ components. The position of the reference substrate points (BENZAL, TOL) and those of the others under inhibition (BENZAL _INH1_2_3, BENZAL_MIX) are shown in Fig. 4 . In the case of inhibition, the points of substrate concentrations on the loading plot have smaller contributions to the first principal component (PC1) than the reference points (BENZAL, BENZAL_SINGLE) without inhibition.
Furthermore, their positions in the second and fourth quadrant approximate to the origin with the decrement of inhibition constant $K_{i}$, which is the increment of the effect of competitive inhibitor (toluene).

\section{Conclusions}

In this study, a new method of detecting inhibition effects of substrate mixtures on badly defined biocatalysts (e.g. commercial mixtures of microorganisms and enzymes) is presented. The method has been confirmed in model as well as experimental data analysis by applying statistical methods. To put it briefly, the normalized concentration data of pollutants are plotted against each other. Without mutual inhibition, a linear relationship is observed. With increasing strength of inhibition, the plots deviate from linearity, and the parameters of the linear regression are changing as follows: the slope is increasing and the intercept is decreasing. The modification of these parameters can be considered as a measure of the inhibition strength. A PCA also proved to be suitable for the detection of the inhibition effects on the efficiency of biocatalyst. The substrate points under inhibition can separate from the reference points on the loading plot of the first two components (PC1, PC2).

Our result showing that toluene inhibits the biodegradation of benzaldehyde is in accordance with the literature (Ferreira et al., 2005; Hess and Antranikian, 2008). If the presented methods can be extended to other pollutant mixtures and further biocatalysts, then the inhibition effects can be identified very easily, which is of great importance for the selection of the most suited biocatalyst for the respective contaminated site with only a few experiments. The novelty of our data evaluation consists of the fact that the correlation between kinetic data series can be very simple and fast. Using our method does not require any specific enzyme kinetic calculating software. However, this approach makes it possible to explore cross-inhibition effects as well. The findings of this study can contribute considerably to the success of the environmental cleaning strategies.

\section{Acknowledgments}

The authors wish to thank Birgit Würz, Karin Lange, and Martina Effenberger (UFZ Helmholtz Centre for Environmental Research, Department of Environmental Microbiology) for skilled technical assistance. The cooperation with two of the authors was supported by the German Federal Environmental Foundation (DBU) under grant no. 30014/529. 


\section{References}

Alcalde M., Ferrer M., Plou J., Ballesteros A. (2006) Environmental biocatalysis: from remediation with enzymes to novel green processes. Trends Biot. 24: 281-287.

Brandt B.W., Kelpin F.D.L., Leeuwen I.M.M.V., Kooijman S.A.L.M. (2004) Modelling microbial adaptation to chan ging availability of substrates. Wat. Res. 38: 1003-1013.

Dana L.D., Bauder J.W. (2011) A general essay on bioremediation of contaminated oil. Montana StateUniversity, Bozeman, Montana, USA.

Dua M., Singh A., Sethunathan N., Johri A. (2002) Biotechnology and bioremediation: successes and limitations. Appl. Microb. Biot. 59(2-3): 143-152.

Esbensen K.H., Guyot D., Westad F., Houmoller L.P. (2002) Multivariate data analysis - in practice: an introduction to multivariate data analysis and experimental design. Camo Process AS.

Ferreira P., Medina M., Guillén F., Martínez M.J., van Berkel W.J.H., Martínez Á.T. (2005) Spectral and catalytic properties of aryl-alcohol oxidase, a fungal flavoenzyme acting on polyunsaturated alcohols. Bioch. J. 389: 731-738.

Harder W., Dijkhuizen L. (1982) Strategies of mixed substrate utilization in microorganisms. Philos. Trans. R. Soc. London B 297:.459-480

Hess M., Antranikian G. (2008) Archaeal alcohol dehydrogenase active at increased temperatures and in the presence of organic solvents. Appl. Microb. Biot. 77: 10031013.
Karigar C.S., Rao S.S. (2011) Role of microbial enzymes in the bioremediation of pollutants: A review. Enzyme Res. 2011: 11.

Lin C.W., Cheng Y.-W., Tsai S.-L. (2007) Multi-substrate biodegradation kinetics of MTBE and BTEX mixtures by Pseudomonas aeruginosa. Proc. Bioch. 42: 1211-1217.

Megharaj M., Ramakrishnan B., Venkateswarlu K., Sethunathan N., Naidu R. (2011) Bioremediation approaches for organic pollutants: A critical perspective. Env. Int. 37: 1362-1375.

Reardon K.F., Mosteller D.C., Bull Rogers J.D. (2000) Biodegradation kinetics of benzene, toluene, and phenol as single and mixed substrates for Pseudomonas putida F1. Biot. Bioeng. 69: 385-400.

Sutherland T., Horne I., Weir K., Coppin C., Williams M., Selleck M., Russell R., Oakeshott J. (2004) Enzymatic bioremediation: from enzyme discovery to applications. Clin. Exp. Pharm. Phys. 31: 817-821.

Trigueros D.E.G., Módenes A.N., Ravagnani M.A.S.S. (2010) Biodegradation kinetics of benzene and toluene as single and mixed substrate: estimation of biokinetics parameters by applying particle swarm optimization. Latin Amer. Appl. Res. 40(3): 219-236.

Vidali M. (2001) Bioremediation - an overview. Pure Appl. Chem. 73(7): 1163-1172. 